74.25.Ha,74.20.-z,74.20.Rp

\title{
Critical field in a model with local pairs
}

\author{
Marcin Mierzejewski and Maciej M. Maśka* \\ Department of Theoretical Physics, Institute of Physics, University of Silesia, 40-007 Katowice, Poland
}

\begin{abstract}
We analyze the role of Zeeman and orbital pair breaking mechanisms in models appropriate for short coherence length superconductors. In particular, we investigate the attractive Hubbard and the pair hoping models. The orbital pair breaking mechanism dominates in the majority of models with $s$-wave and $d$-wave superconducting order parameters. On the other, the repulsive pair hopping interaction leads to $\eta$-type pairing, that is stable against the orbital pair breaking. External magnetic field reduces this type of pairing predominantly due to the Zeeman coupling. According to the recent experiments this mechanism is responsible for closing of the pseudogap. Moreover, the temperature dependence of the gap closing field in $\eta$-phase fits the experimental data very well. We discuss whether the preformed pairs in the $\eta$-phase could be responsible for the pseudogap phenomenon.
\end{abstract}

\section{INTRODUCTION}

Almost twenty years after discovery of hightemperature superconductors (HTSCs) the mechanism responsible for their unusual properties remains unclear. The complex phase diagram of HTSCs suggests that there may be no single mechanism that dominates over the entire doping range. In particular, the normal-state properties in underdoped and overdoped regimes are different. Highly overdoped compounds in the normal state exhibit Fermi liquid behavior, whereas the superconducting state may be described within a weak-coupling BCS theory [1]. On the other hand, in the underdoped regime the HTSCs exhibit unconventional features. The most remarkable of them are the extremely short coherence length and a pseudogap that opens in the normal state. The presence of the pseudogap has been confirmed with the help of various experimental techniques like: angleresolved photoemission [2, 3, 4], intrinsic tunneling spectroscopy [5, [6], NMR [7, [8], infrared [9] and transport [10] measurements. Although, there is no complete theoretical description of the pseudogap, one usually considers this phase as a precursor of the superconductivity. According to this hypothesis formation of Cooper pairs starts at temperature $T^{*}$, higher than the superconducting transition temperature $T_{c}$. Then, at $T_{c}$, these preformed pairs undergo Bose-Einstein condensation.

This hypothesis seems to be supported by recent observations of the vortex-like Nernst signal above $T_{c}$ [11] that evolves smoothly into the analogous signal below the superconducting phase transition [12]. The Meissner effect does not occur in the pseudogap phase due to strong phase fluctuations rather than the vanishing of the superfluid density. Therefore, theoretical description of the suppression of the Meissner effect requires an approach beyond the mean-field level. Despite the absence of the Meissner effect above $T_{c}$, one can observe inhomogeneous

*Electronic address: maciek@phys.us.edu.pl magnetic domains that are interpreted as precursors to the Meissner state [13].

The short coherence length indicates that the pairing takes place in the real space, leading to boson-like objects. A few models are commonly used to describe systems with the local pairs. Namely, the attractive Hubbard (AH) model 14], fermion-boson 15] and purely bosonic models 16], as well as the Penson-Kolb (PK) model[17], i.e., the tight-binging model with local pair hopping. These models should be considered as effective approaches which do not explain the microscopic origin of the pairing interaction.

Another unusual property of HTSCs is related to their behavior in the external magnetic field. In particular, temperature dependence of the upper critical field $H_{c 2}$ has a positive curvature 18, 19] in contradistinction to classical superconductors, where a negative curvature is observed. Moreover, $H_{c 2}$ does not saturate even at genuinely low temperature. Recent experiments 20] show that also the pseudogap is destroyed by sufficiently high magnetic field, $H_{p g}$. Although, the temperature dependence of $H_{p g}$ has a negative curvature, it significantly differs from the predictions of the standard HelfandWerthammer theory [21]. Namely, $H_{p g}(T)$ has a large slope at temperatures close to $T^{*}$ and saturates already at $T \simeq 0.7 T^{*}$. These features may assist in verification of the preformed Cooper pairs hypothesis and, more generally, in choosing the most appropriate model of HTSC.

\section{MODEL}

In the present paper we show that opening of the pseudogap and its dependence on the magnetic field can be described within a model with local pair hopping. Our starting point is the two-dimensional (2D) Penson-Kolb model with the Hamiltonian given by:

$$
H=\sum_{i, j, \sigma} t_{i j} \mathrm{e}^{i \Phi_{i j}} c_{i \sigma}^{\dagger} c_{j \sigma}+\sum_{i \sigma}\left(g \mu_{B} H_{z} \sigma-\mu\right) c_{i \sigma}^{\dagger} c_{i \sigma}
$$




$$
-\frac{1}{2} J \sum_{\langle i, j\rangle} \mathrm{e}^{2 i \Phi_{i j}} c_{i \uparrow}^{\dagger} c_{i \downarrow}^{\dagger} c_{j \downarrow} c_{j \uparrow} .
$$

Here, $c_{i \sigma}^{(\dagger)}$ creates (annihilates) an electron with spin $\sigma$ at site $i, t_{i j}$ is the single electron hopping integral between sites $i$ and $j, \mu$ is the chemical potential and $J$ is the nearest neighbor pair hopping interactions. The external magnetic field perpendicular to the lattice $H_{z}$ shifts the energy levels by $g \mu_{B} H_{z} \sigma$ ( $g$ is the gyromagnetic ratio and $\mu_{B}$ is the Bohr magneton) and modifies the hopping terms. The single electron hopping integral acquires the Peierls factor

$$
\Phi_{i j}=\frac{e}{\hbar c} \int_{\mathbf{R}_{j}}^{\mathbf{R}_{i}} \mathbf{A} \cdot d \mathbf{l}
$$

whereas the phase factor in the pair hopping term is twice larger.

The Penson-Kolb model can be derived from a general microscopic tight-binding Hamiltonian [22], where the Coulomb repulsion may lead to the pair hopping interaction. In such a case $J$ is negative (repulsive PensonKolb model). However, treating the Penson-Kolb model as of a phenomenological nature, we assume $J$ to be an effective parameter, that can be negative as well as positive. It can be understood as a result of renormalization originating, e.g., from electron-phonon coupling [23]. For a nonzero single electron hopping integral $J \rightarrow-J$ is not a symmetry of the PK model [24]. However, superconducting correlations occur in the Penson-Kolb model for attractive pair hopping interaction $(J>0)$ as well as for the repulsive one $(J<0)$, provided that the pair hopping is large enough. The latter case is usually referred to as $\eta$-type pairing. Then, the total momentum of the paired electrons is $\mathbf{Q}=(\pi, \pi)$ and the phase of superconducting order parameter alters from one site to the neighboring one. It has been shown that there is a flux quantization and Meissner effect in this state. 25] Superconductivity survives also in the presence of on-site Coulomb repulsion (Penson-Kolb-Hubbard model), provided that this interaction is not too large 26].

\section{A. Density of states}

At the mean-field level, for $J>0$ one obtains an isotropic superconducting gap, identical to that obtained for AH model. On the other hand, in the case of $\eta$-type pairing $(J<0)$, the density of states is finite for arbitrary energy. However, the density of states at the Fermi level may significantly be suppressed for some dopings. In the simplest case of the nearest neighbor hopping the density of states in the $\eta$-phase is of the form:

$$
\rho(\omega)=\frac{1}{2}\left(1-\frac{\mu}{\tilde{\mu}}\right) \rho_{0}(\omega-\tilde{\mu})+\frac{1}{2}\left(1+\frac{\mu}{\tilde{\mu}}\right) \rho_{0}(\omega+\tilde{\mu}) .
$$

Here, $\tilde{\mu}=\sqrt{\mu^{2}+4|J \Delta|^{2}}, \Delta \equiv(-1)^{i}\left\langle c_{i \downarrow} c_{i \uparrow}\right\rangle$ is the $\eta$ phase order parameter and $\rho_{0}$ is the density of states for

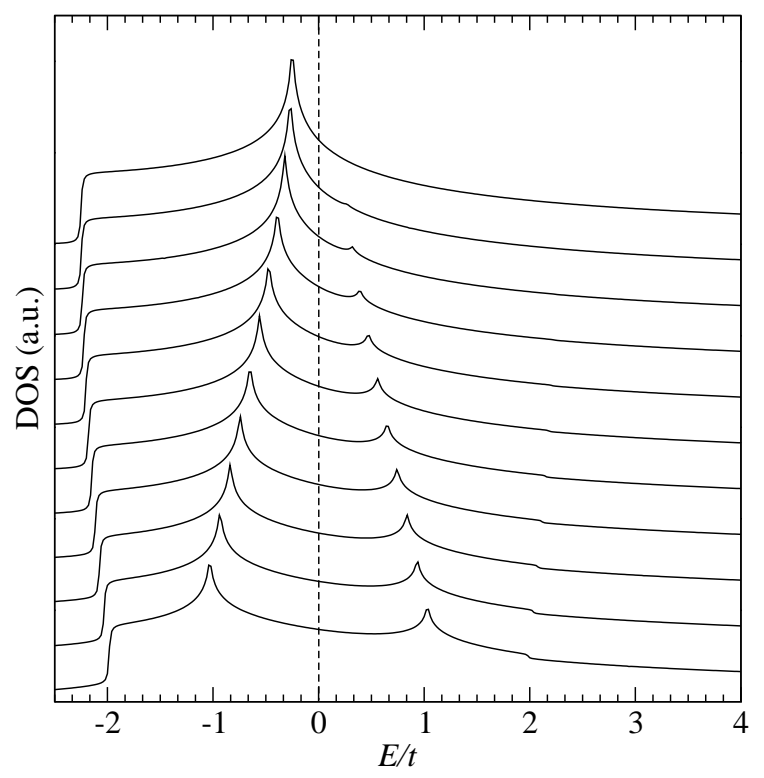

FIG. 1: Density of states for the Penson-Kolb model in the $\eta$-phase. We have used $t^{\prime}=-0.25 t, J=-t$ and $\mu=-0.75 t$. The curves from the topmost to the lowest correspond to the values of the order parameter $\Delta=0,0.1, \ldots, 1$. The dashed line indicates the Fermi level.

$\mu=\Delta=0$. One can see from Eq. (3) that the quasiparticle poles split when $\Delta$ becomes finite. Therefore, a local minimum in the density of states may occur at the Fermi surface. Despite the presence of this minimum the density of states at the Fermi level remains finite provided that $\tilde{\mu}$ is small when compared to the band width. Inclusion of the next nearest neighbor hopping $t^{\prime}$ leads to a more complicated expression for the density of states. However, the structure of $\rho$ in the $\eta$-phase remains unchanged. Fig. 1. shows the density of states calculated for $t^{\prime} \neq 0$ and different values of the $\eta$-phase order parameter. Gradual decreasing of $\rho$ at the Fermi level resembles opening of the pseudogap in HTSCs.

Another feature that could speak in favor of this interpretation is anisotropy of the gap [3]. More precisely, for $t^{\prime} \neq 0$ the magnitude of splitting of the quasiparticle peaks depends on the direction in the Brillouin zone. The splitting of the spectral functions is presented in Fig. 2. As we consider isotropic order parameter the splitting is finite everywhere at the Fermi level, in contradistinction to a purely $d$-wave gap. However, this drawback may be removed when considering a nonlocal pairing.

\section{B. Response to magnetic field}

In contradistinction to the $\mathrm{AH}$ model, the external magnetic field explicitly enters the term responsible for superconductivity, i.e., the pair hopping interaction. Therefore, the differences between AH and PK models may show up in the electromagnetic properties [27. Here, we investigate the temperature dependence 


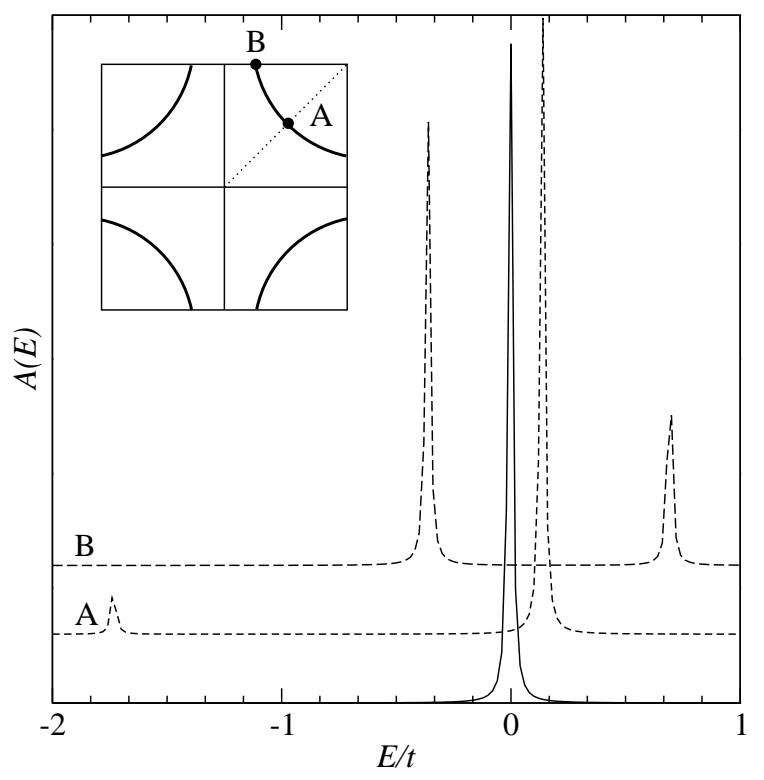

FIG. 2: The spectral functions in various points at the Fermi surface. We have used the same model parameters as in Fig. 1. The position of points $A$ and $B$ is depicted in the inset. The continuous curve corresponds to the case $\Delta=0$, whereas the dashed lines have been obtained for $\Delta=0.5$.

of critical field $H_{\text {crit }}$. It is defined as the highest magnitude of the magnetic field, for that there exists a non-zero solution for the superconducting order parameter:

$$
\Delta_{i}=\left\langle c_{i \downarrow} c_{i \uparrow}\right\rangle .
$$

As we carry out calculations at the mean-field level it is impossible to determine the phase coherence of the Cooper pairs. Therefore, the physical interpretation of the critical field is not unique. In the overdoped regime of cuprates it can directly correspond to the upper critical field. On the other hand, for underdoped systems, and within the precursor scenario of pseudogap, it can be interpreted as $H_{p g}$, i.e., the field at which the incoherent pairs appear. One can argue that the PK model can also be used as an effective model of other superconductors that are characterized by a short coherence length: barium bismuthates, fullerides, Chevrel phases, organic superconductors and heavy fermion systems. 27] In these cases $H_{\text {crit }}$ should be considered as $H_{c 2}$.

For the sake of simplicity we define:

$$
\tilde{\Delta}_{i}=\frac{1}{2} \sum_{j}^{\prime} \mathrm{e}^{2 i \Phi_{i j}} \Delta_{j}
$$

where the prime means that the summation is carried out over the nearest neighbors of site $i$. Then, the mean-field Hamiltonian takes on the following form:

$$
\begin{aligned}
H= & \sum_{i, j, \sigma} t_{i j} \mathrm{e}^{i \Phi_{i j}} c_{i \sigma}^{\dagger} c_{j \sigma}+\sum_{i \sigma}\left(g \mu_{B} H_{z} \sigma-\mu\right) c_{i \sigma}^{\dagger} c_{i \sigma} \\
& -J \sum_{i}\left(c_{i \uparrow}^{\dagger} c_{i \downarrow}^{\dagger} \tilde{\Delta}_{i}+\text { H.c. }\right) .
\end{aligned}
$$

At the mean-field level the only difference between PK and AH models is the presence of $\tilde{\Delta}_{i}$ in Eq. (6) instead of $\Delta_{i}$. Therefore, in order to calculate the critical field one can follow an approach, that has previously been developed for the lattice gas with on-site attraction [28]. Then, one ends up with the lattice version of the Gor'kov equations:

$$
\Delta_{i}=\frac{J}{\beta} \sum_{j, \omega_{n}} \tilde{\Delta}_{j} G\left(i, j, \omega_{n}\right) G\left(i, j,-\omega_{n}\right)
$$

Here, $G\left(i, j, \omega_{n}\right)$ is the one-electron Green's function in the presence of a uniform and static magnetic field. $\omega_{n}$ denotes the fermionic Matsubara frequency. With the help of Eq.(15) one can eliminate $\tilde{\Delta}_{i}$ from the Gor'kov equations. Then, $H_{\text {crit }}(T)$ can be calculated from

$$
\Delta_{i}=\frac{J}{2 \beta} \sum_{\langle j, l\rangle, \omega_{n}} \mathrm{e}^{2 i \Phi_{l j}} \Delta_{j} G\left(i, l, \omega_{n}\right) G\left(i, l,-\omega_{n}\right)
$$

or

$$
\Delta_{i}=\frac{-J}{2 \beta} \sum_{\langle j, l\rangle, \omega_{n}}(-1)^{j+l} \mathrm{e}^{2 i \Phi_{l j}} \Delta_{j} G\left(i, l, \omega_{n}\right) G\left(i, l,-\omega_{n}\right) .
$$

Eqs. (8) and (9) are equivalent since $(-1)^{j+l}=-1$, for the neighboring sites $j$ and $l$. However, it is more convenient to use the first/second of them for attractive/repulsive pair hopping interaction.

In the following we consider only the nearest-neighbor one-particle hopping integral $t$ and use the Landau gauge $\mathbf{A}=H_{z}(0, x, 0)$. Then, the Harper equation

$$
\begin{aligned}
& g\left(\bar{p}_{x}, p_{y}, x+1\right)+2 \cos \left(h x-p_{y} a\right) g\left(\bar{p}_{x}, p_{y}, x\right) \\
& +g\left(\bar{p}_{x}, p_{y}, x-1\right)=t^{-1} E\left(\bar{p}_{x}, p_{y}\right) g\left(\bar{p}_{x}, p_{y}, x\right),
\end{aligned}
$$

determines eigenvalues $E\left(\bar{p}_{x}, p_{y}\right)$ of the one-particle hopping term. The corresponding eigenstates are enumerated by $\bar{p}_{x}, p_{y}$ and are of the form:

$$
U_{x, y}\left(\bar{p}_{x}, p_{y}\right)=e^{i p_{y} y a} g\left(\bar{p}_{x}, p_{y}, x\right) .
$$

Here, $x, y$ are integers which enumerate the lattice sites in $\hat{x}$ and $\hat{y}$ directions, whereas $h /(2 \pi)$ is a ratio of the flux through a lattice cell to one flux quantum. We refer to Ref. 28] for the details.

The one-electron Green's function can be expressed with the help of eigenvalues and eigenstates of the normal-state Hamiltonian. Then, the summation over Matsubara frequencies in Eqs. (8) and (9) can explicitly be carried out. In the Landau gauge the presence of magnetic field does not change the plane-wave behavior in $\hat{y}$-direction [see Eq. (11)]. Therefore, the superconducting order parameter depends only on $x$ and pairing of electrons takes place for the same $\hat{y}$-components of their momenta, as in the absence of magnetic field, i.e., $\left(p_{y},-p_{y}\right)$ for $J>0$ and $\left(p_{y}, \pi-p_{y}\right)$ for $J<0$. Taking 
these features into account one can rewrite the Gor'kov equations for the attractive:

$$
\begin{aligned}
\Delta_{x^{\prime}}= & \frac{J}{2 \sqrt{N}} \sum_{x} \Delta_{x} \sum_{p_{y}, \bar{p}_{x}, \bar{k}_{x}} \chi\left(\bar{p}_{x}, p_{y}, \bar{k}_{x},-p_{y}\right) \\
& \times\left[2 \cos (2 h x) g\left(\bar{p}_{x}, p_{y}, x\right) g\left(\bar{k}_{x},-p_{y}, x\right)\right. \\
& +g\left(\bar{p}_{x}, p_{y}, x+1\right) g\left(\bar{k}_{x},-p_{y}, x+1\right) \\
& \left.+g\left(\bar{p}_{x}, p_{y}, x-1\right) g\left(\bar{k}_{x},-p_{y}, x-1\right)\right] \\
& \times g\left(\bar{p}_{x}, p_{y}, x^{\prime}\right) g\left(\bar{k}_{x},-p_{y}, x^{\prime}\right)
\end{aligned}
$$

as well as for the repulsive pair hopping interaction:

$$
\begin{aligned}
\Pi_{x^{\prime}}= & \frac{-J}{2 \sqrt{N}} \sum_{x} \Pi_{x} \sum_{p_{y}, \bar{p}_{x}, \bar{k}_{x}} \chi\left(\bar{p}_{x}, p_{y}, \bar{k}_{x}, \pi-p_{y}\right) \\
& \times\left[2 \cos (2 h x) g\left(\bar{p}_{x}, p_{y}, x\right) g\left(\bar{k}_{x}, \pi-p_{y}, x\right)\right. \\
& -g\left(\bar{p}_{x}, p_{y}, x+1\right) g\left(\bar{k}_{x}, \pi-p_{y}, x+1\right) \\
& \left.-g\left(\bar{p}_{x}, p_{y}, x-1\right) g\left(\bar{k}_{x}, \pi-p_{y}, x-1\right)\right] \\
& \times g\left(\bar{p}_{x}, p_{y}, x^{\prime}\right) g\left(\bar{k}_{x}, \pi-p_{y}, x^{\prime}\right) .
\end{aligned}
$$

Here $\Pi_{x} \equiv \Delta_{x, y}(-1)^{y}$ and the Cooper pair susceptibility $\chi\left(\bar{p}_{x}, p_{y}, \bar{k}_{x}, k_{y}\right)$ has the following form:

$$
\begin{aligned}
\chi\left(\bar{p}_{x}, p_{y} ; \bar{k}_{x}, k_{y}\right) & =\left[\tanh \frac{E\left(\bar{p}_{x}, p_{y}\right)-\mu-g \mu_{B} H_{z}}{2 k_{B} T}\right. \\
& \left.+\tanh \frac{E\left(\bar{k}_{x}, k_{y}\right)-\mu+g \mu_{B} H_{z}}{2 k_{B} T}\right] \\
& \times\left[2\left(E\left(\bar{p}_{x}, p_{y}\right)+E\left(\bar{k}_{x}, k_{y}\right)-2 \mu\right)\right]^{-1} .
\end{aligned}
$$

The above equations determine the strength of magnetic field at which the local pairing disappears. We have carried out calculations for $150 \times 150$ cluster with periodic boundary conditions (bc) along the $\hat{y}$ axis. As the Landau gauge breaks the translation invariance along $\hat{x}$ axis we have used fixed bc in this direction. Our previous calculations indicate that such a size of cluster is sufficient to obtain convergent results. [28]

Fig. 3 shows the temperature dependence of $H_{\text {crit }}$ obtained for attractive and for the repulsive pair hopping interaction. These results are compared with $H_{\text {crit }}(T)$ calculated from the $2 \mathrm{D}$ AH model 28 with $U=-t$. We have adjusted the strength of the pair hopping interaction to obtain the same critical temperature in the absence of magnetic field. For $J>0 H_{\text {crit }}(T)$ in PK model is very close to that of $\mathrm{AH}$ model. It means that in the case of $s$-wave pairing the Peierls factor in the pair hopping term leads only to a small decrease of superconducting correlations. However, the temperature dependence of $H_{\text {crit }}$ in the $\eta$-state differs qualitatively from the $s$-wave case. Namely, $H_{\text {crit }}(T)$ has a very large slope for a weak magnetic field and saturates already at relatively high temperature. Such a behavior of the critical field resembles $H_{p g}(T)$, that has recently been observed

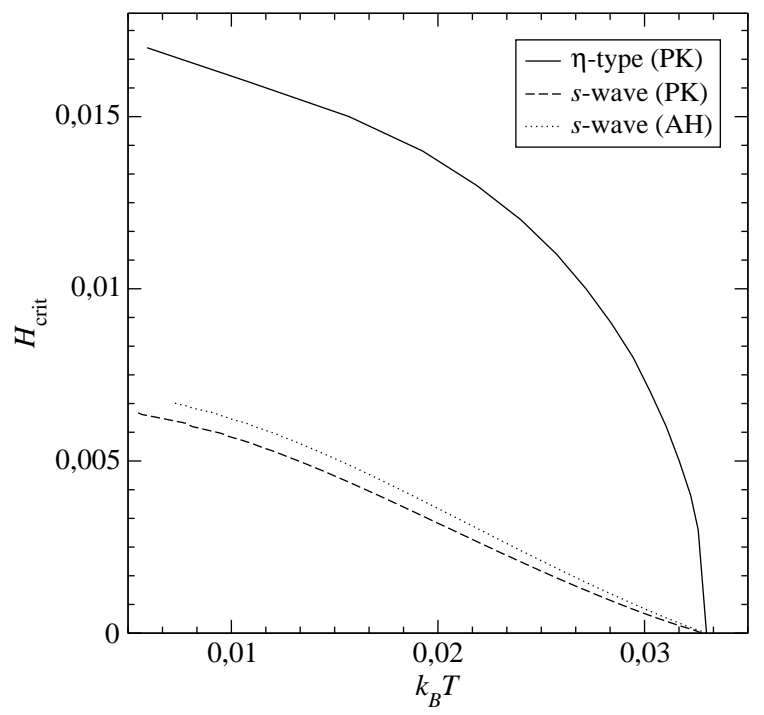

FIG. 3: Temperature dependence of $H_{\text {crit }}$ for $t^{\prime}=0$ and $\mu=0$. Continuous curve has been obtained for the PK model with $J=-1.56 t$ ( $\eta$-type pairing). The dashed line corresponds the attractive pair hopping interaction $J=0.5 t$. The dotted line shows the critical field in the AH model with $U=t$.

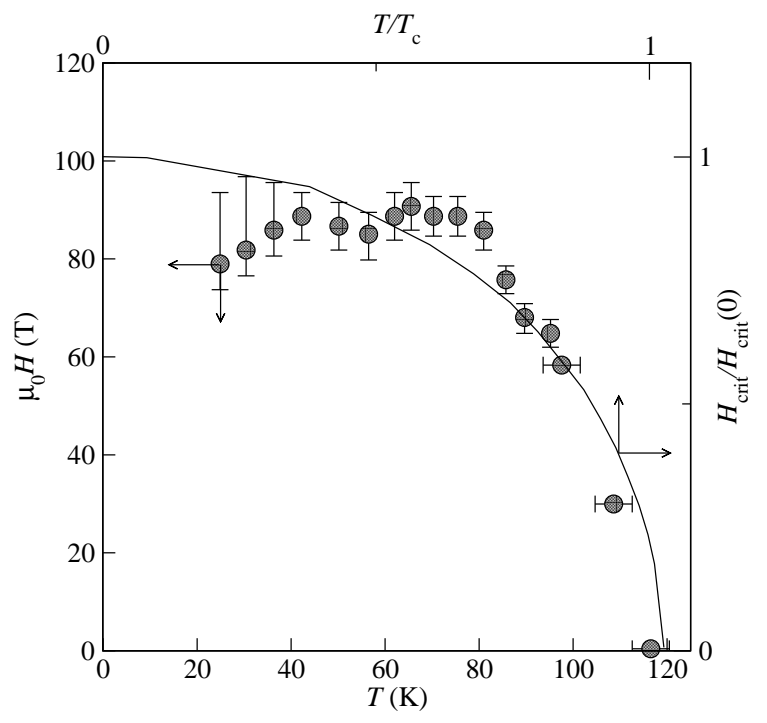

FIG. 4: Fit of the theoretical results to the experimental data for the pseudogap closing field $H_{p g}(T)$. 20] The continuous line represents $H_{\text {crit }}(T)$ calculated for the repulsive pair hopping interaction. We have used the same model parameters as for the $\eta$-pairing in Fig. 3.

in $\mathrm{Bi}_{2} \mathrm{Sr}_{2} \mathrm{CaCu}_{2} \mathrm{O}_{8+\mathrm{y}}$. [20] In Fig. 4 we compare our results and the experimental data.

Within the Helfand-Werthammer theory, the temperature dependence of critical field is predominantly determined by the diamagnetic pair breaking mechanism. The Zeeman coupling becomes important only for suf- 


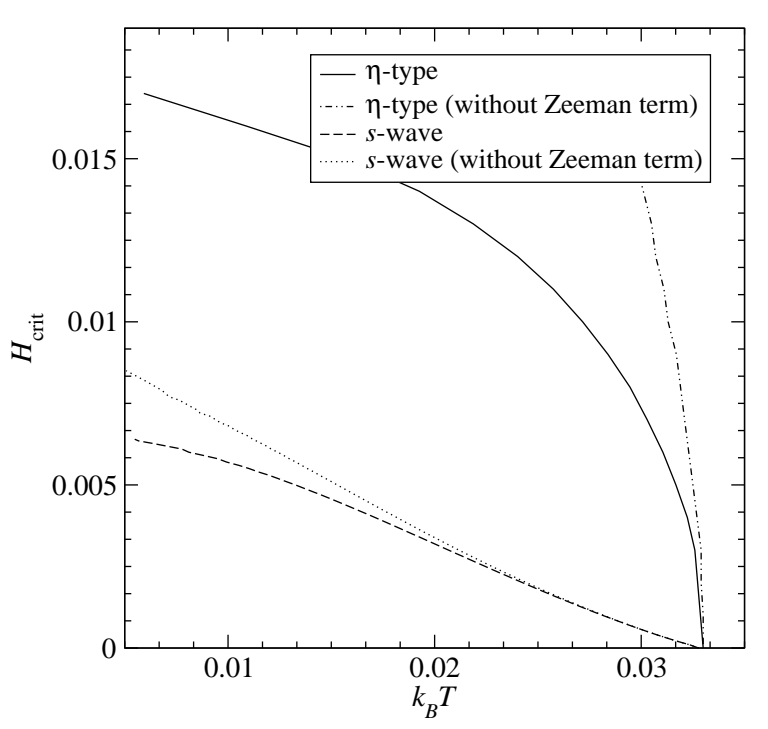

FIG. 5: $H_{\text {crit }}(T)$ calculated for attractive and repulsive PK model with and without the Zeeman coupling. We have used the same model parameters as in Fig. 3.

ficiently strong magnetic field. This feature holds also in the case of the lattice gas. 229] In order to investigate the role of the Zeeman and orbital contributions in PK model, we have repeated our calculations in the absence of the Zeeman term. The resulting $H_{\text {crit }}(T)$ is shown in Fig. 5 .

In contradistinction to the $s$-wave superconductivity, the diamagnetic pair breaking is of minor importance in the case of $\eta$-pairing. This feature is responsible for extremely high values of $H_{\text {crit }}$ in the absence of Zeeman term. Experimental investigations 20] show that the pseudogap closing field scales linearly with $T^{*}$. In Ref. 20] the value of the scaling factor has been interpreted in favor of the Zeeman coupling as a mechanism that closes the pseudogap. This pair breaking mechanism dominates also in the case of $\eta$-pairing.

\section{SUMMARY}

To summarize, we have investigated the PK model with attractive as well as repulsive pair hopping interaction. We have shown that the repulsive pair hopping term may lead to the occurrence of local minimum in the density of states, that is characteristic for pseudogap phase of underdoped cuprates. It originates from the spitting of the quasiparticle peaks. Despite the on-site pairing the magnitude of the splitting is a direction-dependent quantity, provided that $t^{\prime} \neq 0$. Anisotropy of the pseudogap is observed in ARPES experiments. [3] We have also calculated the temperature dependence of $H_{\text {crit }}$, defined as the highest magnetic field for which there exists a non-zero solution for the order parameter. We have found that in the case of $\eta$-type pairing $H_{\text {crit }}(T)$ reproduces the experimental data for the pseudogap closing field. These features do not occur for attractive pair hopping interaction. In this case the gap structure as well as $H_{\text {crit }}(T)$ are similar to those obtained for AH model.

Our approach to the critical field accounts both for Zeeman and diamagnetic pair breaking mechanisms. In the case of $s$-wave pairing inclusion of the Zeeman coupling does not lead to any essential changes in $H_{\text {crit }}(T)$. On the other hand, Zeeman term is of crucial importance for $\eta$-pairing, whereas the diamagnetic pair breaking is ineffective. According to the experimental data the pseudogap is closed by the Zeeman splitting.

As we have previously [28] shown, other models appropriate for short coherence superconductors have ground states (with $s$-wave or $d$-wave symmetry) that are almost insensitive to the Zeeman interaction. Therefore, the PK model with $J<0$ is unique in that the gap is closed predominantly due to the Zeeman interaction.

Collecting the features: the presence of the pseudogap, its anisotropy, Zeeman origin of $H_{\text {crit }}$ (in agreement with the experimental data), the presence of flux quantization and the Meissner effect, (consistent with the preformed Cooper pairs scenario), may lead to a tempting hypothesis that the pair hopping can be responsible for the pseudogap. However, in order to avoid the problem of interpretation of the critical field, it should be verified beyond the mean-field level, discussed in this paper.

\section{Acknowledgments}

We acknowledge a stimulating discussion with S. Robaszkiewicz. This work was supported in part by the Polish State Committee for Scientific Research, Grant No. 2 P03B 05023.
[1] Y. Kubo, Y. Shimakawa, T. Manako, and H. Igarashi, Phys. Rev. B 43, 7875 (1991); M. Suzuki and M. Hikita, Phys. Rev. B 44, 249 (1991); C. Proust, E. Boakin, R. W. Hill, L. Taillefer and A. P. Mackenzie, Phys. Rev. Lett. 89, 147003 (2002).

[2] D. S. Marshall, D. S. Dessau, A. G. Loeser, C.-H. Park, A. Y. Matsuura, J. N. Eckstein, I. Bozovic, P. Fournier, A. Kapitulnik, W. E. Spicer, and Z.-X. Shen, Phys. Rev. Lett. , 76, 4841 (1996).
[3] H. Ding,T. Yokoya, J. C. Campuzano, T. Takahashi, M. Randeria, M. R. Norman, T. Mochiku, K. Hadowaki, and J. Giapintzakis, Nature (London) 382, 51 (1996).

[4] M. R. Norman, H. Ding, M. Randeria, J. C. Campuzano, T. Yokoya, T. Takeuchi, T. Takahashi, T. Mochiku, K. Kadowaki, P. Guptasarma, D. Hinks, Nature (London) 392, 157 (1998).

[5] V. M. Krasnov, A. Yurgens, D. Winkler, P. Delsing, and T. Claeson, Phys. Rev. Lett. 84, 5860 (2000). 
[6] V. M. Krasnov, A. E. Kovalev, A. Yurgens, and D. Winkler, Phys. Rev. Lett. 86, 2657 (2001).

[7] G. V. M. Williams, J. L. Tallon, E. M. Haines, R. Michalak, and R. Dupree, Phys. Rev. Lett. 78, 721 (1997).

[8] G. V. M. Williams, J. L. Tallon, J. W. Quilty, H. J. Trodahl, and N. E. Flower, Phys. Rev. Lett. 80, 337 (1998).

[9] D. N. Basov, T. Timusk, B. Dabrowski, and J. D. Jorgensen, Phys. Rev. B 50, 3511 (1994).

[10] J. L. Tallon, J. R. Cooper, P. S. I. P. N. de Silva, G. V. M. Williams, and J. W. Loram, Phys. Rev. Lett. 75, 4114 (1995).

[11] Z. A. Xu, N. P. Ong, Y. Wang, T. Kakeshita, and S. Uchida, Nature 406, 486 (2000).

[12] Yayu Wang, Z. A. Xu, T. Kakeshita, S. Uchida, S. Ono, Yoichi Ando, and N. P. Ong, Phys. Rev. B64, 224519 (2001).

[13] I. Iguchi, T. Yamaguchi, and A. Sugimoto, Nature 412, 420 (2001).

[14] R. Micnas, J. Ranniger, and S. Robaszkiewicz, Rev. Mod. Phys. 62, 113 (1990).

[15] J. Ranninger and S. Robaszkiewicz, Physica B 135, 468 (1985); S. Robaszkiewicz, R. Micnas, and J. Ranninger, Phys. Rev. B 36, 180 (1987).

[16] A. S. Alexandrov, V. N. Zavaritsky, W. Y. Liang, and P. L. Nevsky, Phys. Rev. Lett. 76, 983 (1996).

[17] K. A. Penson and M. Kolb, Phys. Rev. B 33, 1663 (1986).
[18] M. S. Osofsky, R. J. Soulen, Jr., S. A. Wolf, J. M. Broto, H. Rakoto, J. C. Ousset, G. Coffe, S. Askenazy, P. Pari, I. Bozovic, J. N. Eckstein, and G. F. Virshup, Phys. Rev. Lett. 71, 2315 (1993).

[19] A.P. Mackenzie, S.R. Julian, G.G. Lonzarich, A. Carrington, S.D. Hughes, R.S. Liu, and D.C. Simclair, Phys. Rev. Lett. 71, 1238 (1993).

[20] T. Shibauchi, L. Krusin-Elbaum, Ming Li, M. P. Maley, and P. H. Kes, Phys. Rev. Lett. 86, 5763 (2001).

[21] E. Helfand and N. R. Werthammer, Phys. Rev. Lett. 13, 686 (1964); Phys. Rev. 147, 288 (1966).

[22] J. Hubbard, Proc. R. Soc. London, Ser. A 276, 238 (1963).

[23] S. Robaszkiewicz and B. R. Bułka, Phys. Rev. B 59, 6430 (1999).

[24] G. I. Japaridze, A. P. Kampf, M. Sekania, P. Kakashvili, and Ph. Brune, Phys. Rev. B 65, 014518 (2001).

[25] C. N. Yang, Phys. Rev. Lett. 63, 2144 (1989); C. N. Yang and S. Zhang, Mod. Phys. Lett. B 4, 759 (1990).

[26] F, Dolcini and A. Montorsi, Phys. Rev. B 62, 2315 (200).

[27] W. R. Czart and S. Robaszkiewicz, Phys. Rev. B 64, 104511 (2001).

[28] M. Mierzejewski and M. M. Maśka, Phys. Rev. B 60, 6300 (1999); ibid. 66, 214527 (2002); M. M. Maśka and M. Mierzejewski, ibid. 64, 064501 (2001).

[29] M. M. Maśka, Phys. Rev. B 66, 054533 (2002). 